\title{
Diurnal anisotropy enhancement due to non-local effects of coronal mass ejections
}

\section{Nutthawara Buatthaisong, ${ }^{a}$ David Ruffolo, ${ }^{a, *}$ Alejandro Sáiz, ${ }^{a}$ Chanoknan Banglieng, ${ }^{b}$ Warit Mitthumsiri, ${ }^{a}$ Tanin Nutaro ${ }^{c}$ and Waraporn Nuntiyakul ${ }^{d}$}

${ }^{a}$ Department of Physics, Faculty of Science, Mahidol University, Bangkok 10400, Thailand

${ }^{b}$ Division of Physics, Faculty of Science and Technology, Rajamangala University of Technology Thanyaburi, Pathum Thani 12110, Thailand

${ }^{c}$ Department of Physics, Faculty of Science, Ubon Ratchathani University, Ubon Ratchathani 34190, Thailand

${ }^{d}$ Department of Physics and Materials Science, Faculty of Science, Chiang Mai University, Chiang Mai 50200, Thailand

E-mail: david.ruf@mahidol.ac.th

In addition to solar modulation according to the $\sim 11$-year sunspot cycle and the $\sim 22$-year solar magnetic cycle, the time profile of the Galactic cosmic ray flux can also exhibit short-term ( 2-week) modulation events. These are distinct from Forbush decreases in that they are more symmetric in time and evolve over a time scale much longer than the transit of an interplanetary shock and/or coronal mass ejection (CME). Using data from the Princess Sirindhorn Neutron Monitor at the summit of Doi Inthanon, Thailand, with the world's highest effective vertical geomagnetic cutoff rigidity for a fixed station $(16.7 \mathrm{GV})$, we have examined the solar diurnal anisotropy and find that it exhibited strong peaks during two short-term modulation events in 2012, which were indeed stronger than the diurnal anisotropy variation from sunspot minimum to maximum. We attribute these short-term modulation events to non-local effects of CME shocks. CME shocks (possibly single, multiple, or merged) that propagate beyond Earth inhibit the access of cosmic rays for $\sim 2$ weeks. The direction of anisotropy enhancement favors an explanation in terms of cosmic ray diffusion perpendicular to the interplanetary magnetic field, which eventually causes the cosmic ray flux to stop decreasing and gradually recover.

$37^{\text {th }}$ International Cosmic Ray Conference (ICRC 2021)

July 12th - 23rd, 2021

Online - Berlin, Germany

\footnotetext{
${ }^{*}$ Presenter
} 


\section{Introduction}

The flux of GCRs shows variations with the $\sim 11$-year sunspot cycle and $\sim 22$-year solar magnetic cycle, a process is known as solar modulation. During high solar activity, the flux of GCRs tends to decline, while it rises higher at near sunspot minimum. In addition, near solar minimum, the GCR profile may be either peak-like or plateau-like [1], for negative or positive solar magnetic polarity, respectively, in the solar magnetic cycle with a $\sim 22$-year period. When the magnetic field points outward over the northern solar hemisphere and inward over the southern hemisphere, it is called an A > 0 solar magnetic epoch. On the other hand, when the magnetic field points outward over the south and inward over the north of the solar hemisphere, this is called an $\mathrm{A}<0$ solar magnetic epoch.

Apart from variation due to the solar cycle and solar magnetic cycle, solar activity affects the short-term variations of GCRs, especially during solar maximum. An example of a short-term event is a Forbush decrease (FD), a transient decreases of CR flux due to the passage by the Earth of a coronal mass ejections (CME) - an explosion of plasma and magnetic field from the Sun's corona - and/or its associated shock. In general, FDs show a count rate decrease over hours or days [2]. From Mangeard et al. (2018) [3], the GCR flux decrease from a Forbush decrease is often negligible when the count rate is averaged over Carrington rotation (CarRot) periods of $\sim 27$ days. However, they suggested that several short-term modulation features were found even when they excluded time periods with strong FDs. The events lasted $1-2$ CarRots. They proposed that these were possibly related to CMEs that did not pass directly to the Earth. Here we show that non-local effects of the CMEs and associated shocks, which propagate outward from the Sun and beyond the Earth, can affect the count rate and anisotropy detected by neutron monitors on Earth.

Several studies reported the relationship between FDs and diurnal anisotropy of GCs. The diurnal anisotropy is defined as a short-term variation in CR flux with a periodicity of 24 hours as the Earth rotates. The asymptotic direction and flow of GCRs can be inferred from the phase and amplitude of the anisotropy. FDs can be associated with diurnal anisotropy enhancement [4-6]. The enhancement of anisotropy might start before a FD event by a couple of days [7].

We use data from the Princess Sirindhorn Neutron Monitor (PSNM), the station with the world's highest effective vertical cutoff rigidity $(16.7 \mathrm{GV})$, which means that we can investigate relatively high energy cosmic ray ions. The PSNM station has operated since the end of 2007 and provided continuous information on the recent sunspot cycle. Here we utilize the data to investigate GCR variation through the harmonic analysis of diurnal anisotropy based on neutron count rate to understand the relation between GCR anisotropy and solar activity.

\section{Data and Correction}

\subsection{PSNM Data}

Cosmic ray data in this study were mostly obtained from PSNM. The station is located at the summit of Doi Inthanon, Thailand's highest mountain at $18.59^{\circ} \mathrm{N}$ and $98.49^{\circ} \mathrm{E}$ and altitude 2,560 $\mathrm{m}$. The hourly data are available from http://www. thaispaceweather.com/, including data from 2007 - 2021. We extracted the data, including the pressure corrected and uncorrected data, local pressure, the central detected time, and the day of the year between 2008 to 2019. In addition, 
for specific time periods we also investigated cosmic ray data from the Mawson NM in Antarctica, with an atmosphere-limited cutoff of $\sim 1 \mathrm{GV}$.

Neutron monitor count rates must be corrected for pressure variation because of the high sensitivity to the airmass as neutrons lose energy while travelling through the atmosphere. Subsequently, the data were corrected for the effect of precipitable water vapor (PWV). Water vapor in the local atmosphere significantly affects the count rate detected by PSNM. According to the location of PSNM, the value of precipitable water vapor is highly variable because of the rainy season, roughly from May through November. We apply triangular smoothing over \pm 5 days to the PWV derived from the Global Atmospheric Data Assimilation (GDAS) database and a fitting parameter from [8] to obtain the pressure- and vapor-corrected count rate

$$
C_{P W V}=\frac{C_{p}\left(1-a P W V_{0}\right)}{1-a P W V},
$$

where $C_{p}$ is the hourly pressure corrected count rate, $a=(10.18 \pm 2.4) \times 10^{-5} \mathrm{~mm}^{-1}$ is the negative of the linear fitting slope, $P W V_{0}$ is the median value of PWV in the dry season at PSNM during $2008-2018$, and $P W V$ is the hourly PWV value.

Due to the Earth's rotation, neutron monitors are able to detect daily variations of GCR associated with the so-called diurnal anisotropy (DA) of the GCR directional distribution. We could find the $1^{\text {st }}$-order GCR anisotropy from PSNM data using a $1^{\text {st }}$-order harmonic analysis of the excess over a 24-hour running average of the corrected neutron count rate. The excess was fitted to a sinusoidal curve at a 24-hour period in universal time (UT), with a cosine amplitude $D_{x}$ and sine amplitude $D_{y}$. Generally, since the primary cosmic rays (CRs) are charged particles, the Earth's magnetic field can bend their trajectories. Then we have to apply a matrix to correct the data for geomagnetic bending as

$$
\left(\begin{array}{l}
A_{x} \\
A_{y}
\end{array}\right)=\frac{1}{\left(c_{1}^{1}\right)^{2}+\left(s_{1}^{1}\right)^{2}}\left[\begin{array}{cc}
c_{1}^{1} & -s_{1}^{1} \\
s_{1}^{1} & c_{1}^{1}
\end{array}\right]\left(\begin{array}{l}
D_{x} \\
D_{y}
\end{array}\right),
$$

where $A_{x}$ and $A_{y}$ are components of the anisotropy in UT after correction by the coupling coefficients $c_{1}^{1}$ and $s_{1}^{1}$ which, for PSNM, are 0.3078 and 0.5920 , respectively (T. Kuwabara, private communication, 2010). Then, this anisotropy vector is rotated to local time (LT) by another matrix:

$$
\left(\begin{array}{l}
S_{x} \\
S_{y}
\end{array}\right)=\left[\begin{array}{cc}
\cos (\lambda) & -\sin (\lambda) \\
\sin (\lambda) & \cos (\lambda)
\end{array}\right]\left(\begin{array}{l}
A_{x} \\
A_{y}
\end{array}\right),
$$

where $\lambda$ is the longitude of PSNM $\left(98.49^{\circ}\right)$. Now $S_{x}$ is the equatorial component of the $1^{\text {st }}$-order GCR anisotropy (flow) toward local noon, $12 \mathrm{~h} \mathrm{LT}$, which roughly corresponds to the $x$-direction in geocentric solar ecliptic (GSE) coordinates. Similarly, $S_{y}$ is the equatorial component toward the dusk sector, $18 \mathrm{~h} \mathrm{LT}$, which roughly corresponds to the GSE $y$-direction.

At the high cutoff rigidity of PSNM, the diurnal anisotropy is quite small $(\leq 1 \%)$, and to reduce statistical uncertainty we took 7-day averages of $S_{x}$ and $S_{y}$ for scientific interpretation. Then we obtain the diurnal anisotropy amplitude (DA $\equiv \sqrt{S_{x}^{2}+S_{y}^{2}}$ ) and phase (LT of maximum GCR flux) from the components $S_{x}$ and $S_{y}$. Note that when DA (the magnitude of the anisotropy vector) is temporarily less than $\sim 0.1 \%$, the GCR flow is nearly zero and the phase is not really meaningful for indicating a GCR flow direction. 


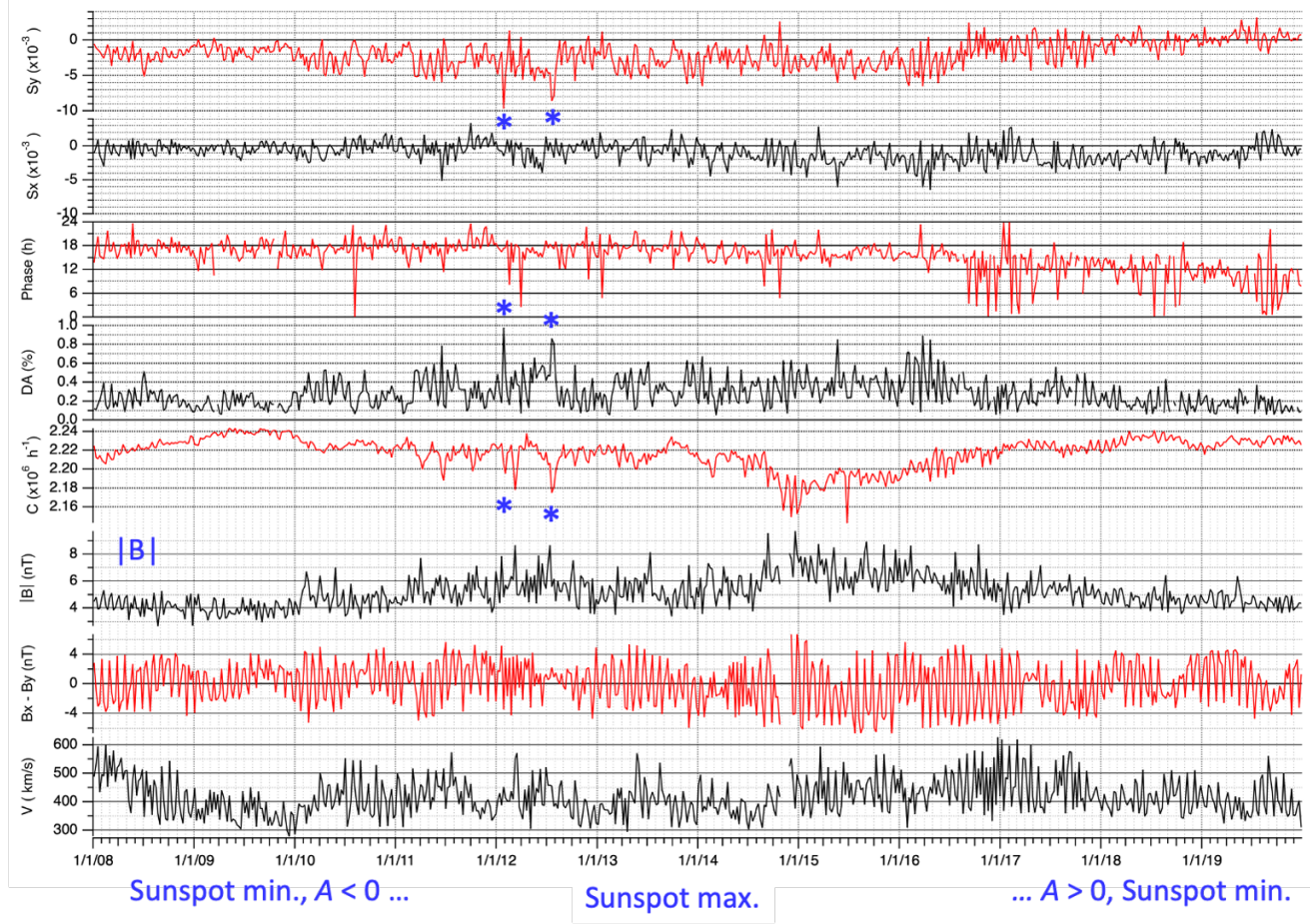

Figure 1: Doi Inthanon NM diurnal anisotropy and count rate, and solar wind parameters, 2008-2019 (7-day averages).

\subsection{Solar Wind Data}

We also considered solar wind information to characterize the short-term solar modulation events and find the relation between GCRs and solar activity. The 1-minute resolution data of solar wind magnetic field and flow speed were from OmniWeb, https: //omniweb.gsfc.nasa.gov/, as observed by NASA's Wind spacecraft.

Initially, the data included magnetic field components (nT) and solar wind flow speed $(\mathrm{km} / \mathrm{s})$. Subsequently, we utilized the differences of the magnetic field in the GSE $x$ and $y$ directions. The difference, $B_{x}-B_{y}$, helps to indicate solar magnetic sectors, which affect the gradient anisotropy. In greater detail, the interplanetary magnetic field (IMF) line is typically angled $\approx 45^{\circ}$ from the radial $(-x)$ direction, toward $+y$, with the field directed either toward or away from the Sun [9]. Then, magnetic sectors are described by the magnetic field components along the GSE $x$ - and $y$-coordinates. When the $x$-component is larger than the $y$-component, the IMF is directed toward the Sun and vice versa. Additionally, we considered data of magnetic field fluctuation $(d B)$, where $d B^{2}$ is the total variance of the fluctuation in all components.

\section{Results}

Figure 1 shows the 7-day averaged data from 2008 to 2019. Looking at the information in greater detail, we could see that anisotropy fluctuated mostly along the $y$-direction. On top of that, when $\mathrm{A}>0$, the phase was close to 18 hours and gradually decreased to 12 hours when $\mathrm{A}<0$ after 


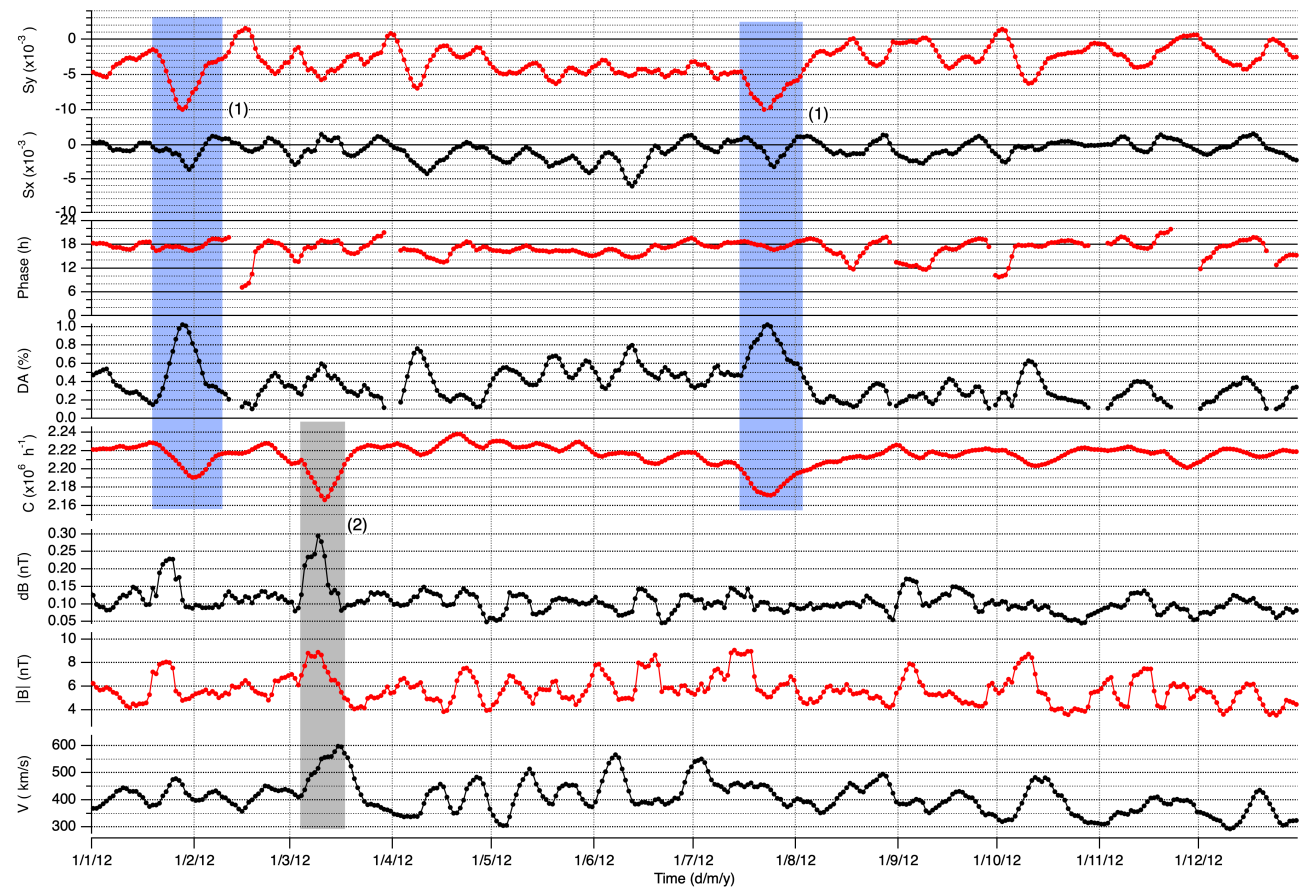

Figure 2: Doi Inthanon NM diurnal anisotropy count rate, and solar wind parameters, 2012 (7-day averages at 1-day cadence): (1) the short-term modulation events, (2) Forbush decrease.

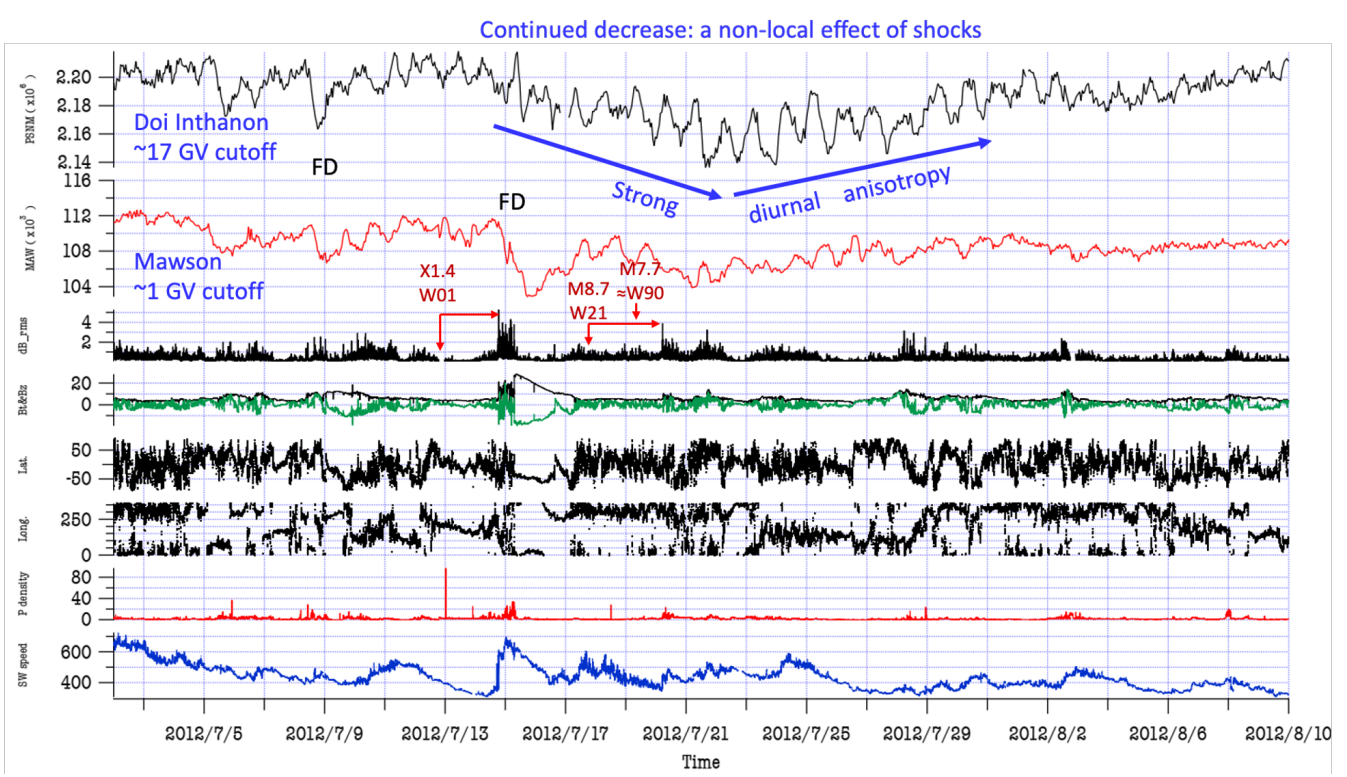

Figure 3: Hourly count rate at Doi Inthanon and Mawson NMs and solar wind data for 2012 Jul 3 to Aug 10. 


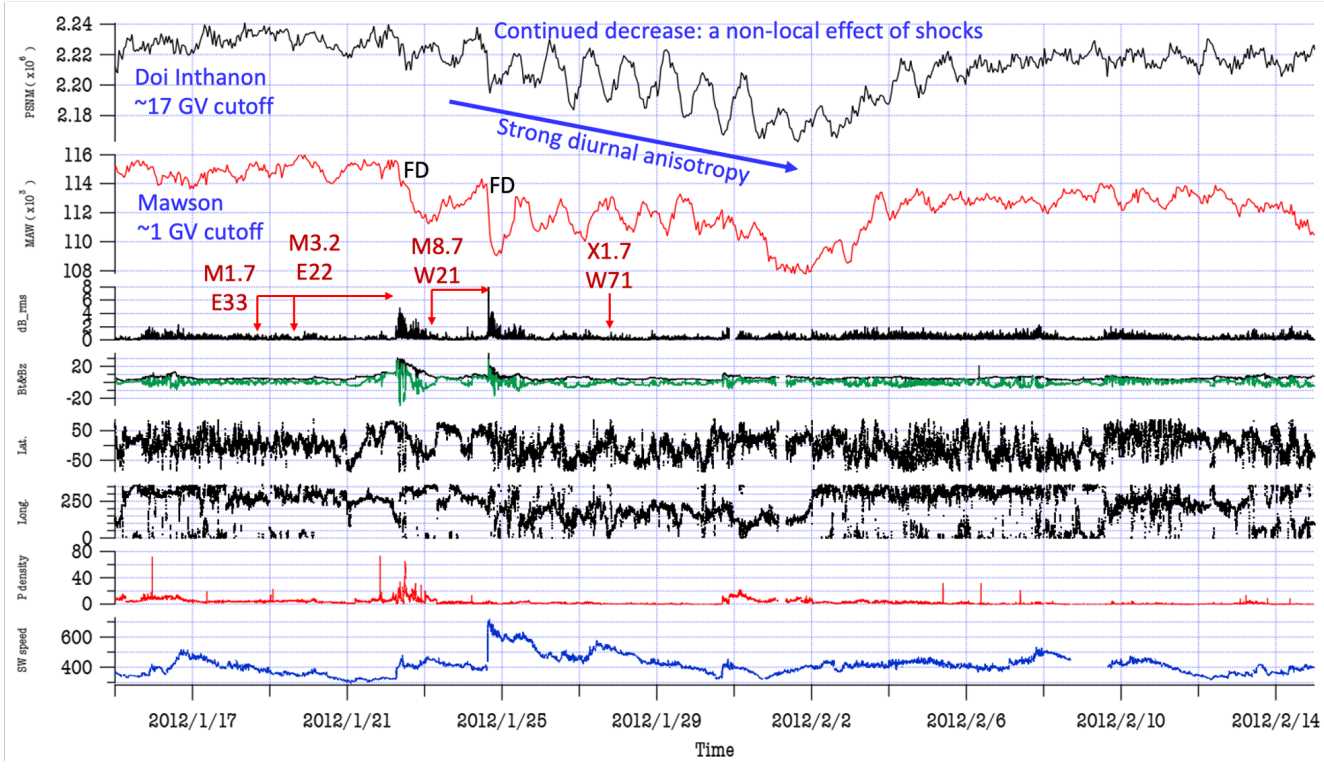

Figure 4: Hourly count rate at Doi Inthanon and Mawson NMs and solar wind data for 2012 Jan 15 - Feb 15.

the solar magnetic polarity reversal of $2012-2014$ (near solar maximum). It should be noted that the 12-hour direction (local noon) points towards the Sun, while the 0-hour direction points to the opposite direction.

Figure 1 shows effects of the sunspot cycle, FDs, short-term modulation events, and 27-day (magnetic sector) variations throughout the period. The magnitude difference $\left(B_{x}-B_{y}\right)$ indicates the solar magnetic sectors, and when the anisotropy reverses along with sector reversals, that is a sign of a gradient anisotropy. During solar maximum in 2012, there were two short-term modulation events with remarkably high anisotropy magnitude in January and July as indicated by “*” symbols in Figure 1 and blue highlighting in Figure 2. Both events exhibited a two-week, roughly symmetric decrease in count rate and sharp peaks of diurnal anisotropy magnitude. An illustration of this is that the solar wind speed, which normally spikes near the start of a Forbush decrease, showed only a slight change in January and was almost constant in July 2012. However, changes in magnetic fluctuation and magnetic field magnitude occurred shortly before these events.

Subsequently, Figures 3 and 4 show 1-hour-averaged data from PSNM and the Mawson Neutron Monitor, where there is only $\sim 1 \mathrm{GV}$ cutoff rigidity, during two periods from January 15 - February 15 and July 3 to August 10 in 2012. It is readily seen from the plots that both cases show strong diurnal anisotropy. However, the data from Mawson were also affected by other phenomena, including a couple of FDs that occurred shortly before the modulation events. In both Figures 3 and 4, there were abrupt changes (e.g., magnetic fluctuation and solar wind speed) when CME shocks passed the Earth during the FDs. On the other hand, PSNM did not clearly observe Forbush decreases when the two short-term events occurred. Later, the count rate gradually decreased and recovered over two weeks.

Since PSNM has a high cutoff rigidity, the station is less sensitive to small structures such as CME ejecta but still can detect large structures, such as interplanetary shocks. We suggest that the 


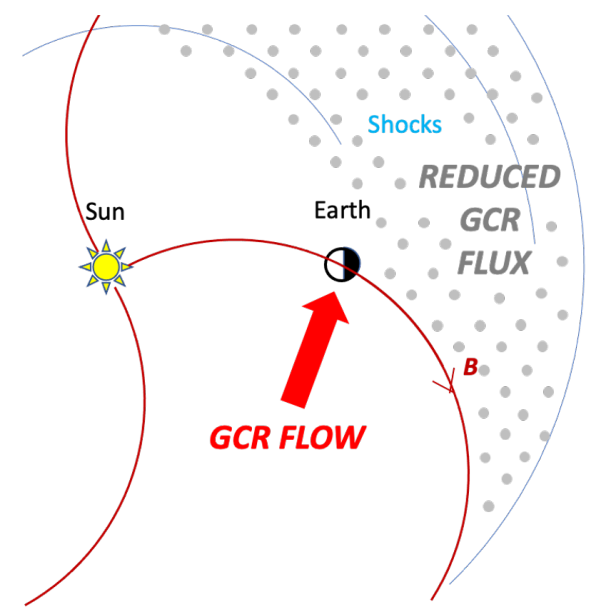

Figure 5: The flow of GCRs along the $-y$ direction due to diffusion perpendicular to the magnetic field as CME-driven interplanetary shocks beyond the Earth inhibit the GCR access via parallel transport.

events relate to the inhibition of GCR access to Earth, by transport parallel to the IMF, due to CMEs and interplanetary shocks that propagated beyond the Earth as shown in Figure 5. As a result, the flux of GCRs measured on the Earth decreased. Thus, the gradual decrease in count rate and enhancement of diurnal anisotropy can be explained by the non-local effects of CME shocks. This is in contrast to FDs, which exhibit a local effect in the sense that the decrease is directly associated with the local passage of a shock, sheath region, and/or CME.

Furthermore, we did not see a significant change of phase (direction) of the anisotropy, and the anisotropy changed mainly in the $-y$ direction. However, the anisotropy enhancement did not reverse during magnetic sector crossings. The best explanation for an anisotropy enhancement that does not reverse with a magnetic reversal, and is associated with a relatively long time scale, is an anisotropy due to diffusion perpendicular to the magnetic field. We propose that the enhanced anisotropy results from a diffusive cross-field inflow of GCR along the $-y$ direction that results in the eventual recovery of the GCR density (Figure 5). We can very roughly estimate a mean free path associated with such perpendicular diffusion as $\lambda_{\perp} \sim 10^{-3} \mathrm{AU}$, in comparison with $\lambda_{\perp} \approx 3 \times 10^{-3}$ $\mathrm{AU}$ as estimated from the theoretical and computational results of [10].

\section{Conclusions}

We examine the solar diurnal anisotropy of GCRs from the Princess Sirindhorn Neutron Monitor (PSNM), Thailand, over a solar 12-year period, from 2008 to 2019. From the time profile of the GCR anisotropy and flux, we found effects from solar modulation and short-term effects, which are related to gradient anisotropy, Forbush decreases, and short-term modulation events. During solar maximum around 2012, there were two short-term modulation events with remarkably high anisotropy magnitude in January and July and symmetric, two-week decreases in neutron count rate. We can conclude that they were non-local effects of CME shocks, in which diffusive cross-field inflow lead to the gradual recovery of the GCR flux. 


\section{Acknowledgements}

N.B. would like to express her sincere gratitude to the Development and Promotion of Science and Technology Talents Project (DPST) for the scholarship and all the opportunities. We acknowledge logistical support to operate the Mawson Neutron Monitor from Australia's Antarctic Program and support via grant RTA6280002 from Thailand Science Research and Innovation.

\section{References}

[1] J.R. Jokipii and B. Thomas, Effects of drift on the transport of cosmic rays. IV - Modulation by a wavy interplanetary current sheet, Astrophys. J. 243 (1981) 1115.

[2] S.E. Forbush, On the effects in cosmic-ray intensity observed during the recent magnetic storm, Phys. Rev. 51 (1937) 1108.

[3] P.-S. Mangeard, J. Clem, P. Evenson, R. Pyle, W. Mitthumsiri, D. Ruffolo et al., Distinct Pattern of Solar Modulation of Galactic Cosmic Rays above a High Geomagnetic Cutoff Rigidity, Astrophys. J. 858 (2018) 43.

[4] G.C. Castagnoli and M.A. Dodero, Trains of diurnal waves in the muonic component at 70 mwe during cosmic-ray decreases, J. Geophys. Res. 74 (1969) 2414.

[5] U. Tortermpun, D. Ruffolo and J.W. Bieber, Galactic Cosmic-Ray Anistropy During the Forbush Decrease Starting 2013 April 13, Astrophys. J. Lett. 852 (2018) L26.

[6] O. Okike, Amplitude of the Usual Cosmic Ray Diurnal and Enhanced Anisotropies: Implications for the Observed Magnitude, Timing, and Ranking of Forbush Decreases, Astrophys. J. 915 (2021) 60.

[7] K. Nagashima, K. Fujimoto, S. Sakakibara, I. Morishita and R. Tatsuoka, Local-time-dependent pre-IMF-shock decrease and post-shock increase of cosmic rays, produced respectively by their IMF-collimated outward and inward flows across the shock responsible for forbush decrease, Planet. Space Sci. 40 (1992) 1109.

[8] C. Banglieng, H. Janthaloet, D. Ruffolo, A. Sáiz, W. Mitthumsiri, P. Muangha et al., Tracking Cosmic-Ray Spectral Variation during 2007-2018 Using Neutron Monitor Time-delay Measurements, Astrophys. J. 890 (2020) 21.

[9] E.N. Parker, Dynamics of the Interplanetary Gas and Magnetic Fields, Astrophys. J. 128 (1958) 664.

[10] D. Ruffolo, T. Pianpanit, W.H. Matthaeus and P. Chuychai, Random Ballistic Interpretation of Nonlinear Guiding Center Theory, Astrophys. J. Lett. 747 (2012) L34. 